\title{
Aprendizagem Observacional em Crianças com Autismo: Efeitos do Ensino de Respostas de Monitoramento via Videomodelação
}

\author{
Izabel Cristina da Silva Brasilense ${ }^{1, *}$, Eileen Pfeiffer Flores², \\ Romariz da Silva Barros ${ }^{1,3}$ \& Carlos Barbosa Alves de Souza ${ }^{1,3}$ \\ 'Universidade Federal do Pará, Belém, PA, Brasil \\ ${ }^{2}$ Universidade de Brasília, Brasilia, DF, Brasil \\ ${ }^{3}$ Instituto Nacional de Ciência e Tecnologia sobre Comportamento, Cognição e Ensino, São Carlos, SP, Brasil
}

\begin{abstract}
RESUMO - O presente estudo investigou o estabelecimento de respostas de monitoramento via videomodelação em duas crianças com transtorno do espectro autista e se essas respostas favoreceriam a aprendizagem observacional de tatos e textuais nessas crianças. Foram realizados pré e pós-testes de aprendizagem observacional via videomodelação. $\mathrm{O}$ treino de monitoramento foi realizado em três etapas: (1) ensino da resposta de monitoramento; (2) desenvolvimento de controle do responder do participante pelo responder do modelo e (3) controle do responder do participante pela consequência para o modelo. As duas crianças aprenderam a resposta de monitoramento em quatro sessões e houve a aquisição de tatos e textuais por aprendizagem observacional para um dos participantes. Discute-se o potencial da videomodelação para treinar comportamentos precorrentes necessários para a aprendizagem observacional.

PALAVRAS-CHAVE: aprendizagem observacional, videomodelação, monitoramento, autismo, crianças
\end{abstract}

\section{Observational Learning in Children with Autism: Effects of Teaching Monitoring Responses via Video Modeling}

\begin{abstract}
This study investigated the establishment of monitoring responses via video modeling of two children with autism spectrum disorder and whether those responses stimulate observational learning of tacts and textual behavior for these children. Pre-and post-tests of observational learning via video modeling were performed. Then, monitoring responses were trained in three steps: (1) teaching of monitoring responses; (2) establishment of stimulus control by the model's behavior and (3) establishment of stimulus control by the consequences of the model's behavior. The two participants acquired monitoring responses in four sessions. One participant acquired tacts and textual behavior via observational learning. We discuss the potential of video modeling as a tool to train precurrent behavior necessary for observational learning.

KEYWORDS: observational learning, video modeling, monitoring, autism, children
\end{abstract}

Segundo o Diagnostic and Statistical Manual of Mental Disorders V (DSM-V; American Psychiatric Association, 2013), o Transtorno do Espectro Autista é uma alteração do desenvolvimento caracterizada por déficits constantes na interação social e comunicação e por padrões comportamentais repetitivos e interesses restritos. As dificuldades de interação social podem comprometer a capacidade da criança de ficar sob controle do comportamento de outras pessoas, de imitá-las, o que prejudicaria a capacidade de aprender observando o que os outros fazem (Greer, Dudek-Singer, \& Gautreaux, 2006; Taylor \& DeQuinzio, 2012).

A aprendizagem observacional pode ser caracterizada como aquela que ocorre por meio da observação do comportamento de outros e das consequências que se seguem a esses comportamentos (Bandura, 1969/1979; Fryling, Johnston, \& Hayes, 2011; Greer et al., 2006; Plavnick \& Hume, 2014). Esse tipo de aprendizagem é importante na aquisição de repertórios culturalmente transmitidos, tais como as práticas educacionais formais (Leaf et al., 2012; Plavnick \& Hume, 2014; Taylor \& DeQuinzio, 2012). Além

*E-mail: belbrasiliense@gmail.com 
disso, quando estabelecida como repertório generalizado, a aprendizagem observacional possibilita a aquisição de uma gama ampla de repertórios sem treino direto, caracterizandose como uma cúspide comportamental (do inglês behavioral cusp; Greer et al., 2006; Rosales-Ruiz \& Baer, 1997).

Apesar da dificuldade para aprender por observação, indivíduos com autismo têm se beneficiado de procedimentos que usam vídeos para ensinar diversos repertórios (Ayres \& Langone, 2005; Charlop, Dennis, Carpenter, \& Greenberg, 2010; Delano, 2007; McCoy \& Hermansen, 2007; Nadel et al., 2011; Nikopoulos \& Keenan, 2007; Plavnick \& Ferreri, 2011). A videomodelação tem sido efetiva em treinos de respostas afetivas adequadas (simpatia, apreciação, desaprovação), em situações de jogos de faz-de-conta (Gena, Couloura, \& Kymissis, 2005), ensino de sequências de comportamentos de iniciação social e brincadeira recíproca (Nikopoulos \& Keenan, 2007), ensino de comportamentos socialmente expressivos - comentários verbais, entonação, gestos e expressão facial (Charlop et al. 2010) e aquisição e generalização de mandos (Plavnick \& Ferreri, 2011).

A videomodelação parece ser mais eficaz e eficiente para ensinar crianças com autismo, em comparação com procedimentos "ao vivo" de aprendizagem observacional (Charlop-Christy, Le \& Freeman, 2000). Essa maior eficácia e eficiência pode estar relacionada: (1) à possibilidade de variar de forma sistemática os exemplares de estímulos e ambientes relacionados com o comportamento que se está buscando ensinar, favorecendo, assim, a manutenção e generalização; (2) a um maior controle do procedimento de modelação, que pode ser apresentado em etapas programadas; (3) à repetição exata da mesma situação a ser observada (mesmo modelo e procedimento); (4) à possibilidade de enfatizar o controle discriminativo por meio de repetições e/ou edições do vídeo e (5) a uma possível redução na aversividade da interação social, pois o contato com modelos sociais pode ocorrer, inicialmente, sem o contato face a face (Bellini \& Akullian, 2007; Charlop-Christy et al., 2000; Corbett \& Abdullah, 2005; Nadel et al., 2011).

Uma limitação importante nos estudos sobre aprendizagem observacional (seja em situações 'ao vivo' ou por videomodelação) é que praticamente todos investigam a aprendizagem observacional como variável independente (Plavnick \& Hume, 2014). Ou seja, a aprendizagem observacional é a variável manipulada para verificar seu efeito na aquisição de diferentes repertórios (as variáveis dependentes). Foram localizados apenas três estudos que avaliaram de forma explícita a aprendizagem observacional como variável dependente (DeQuinzio \& Taylor, 2015; Pereira-Delgado \& Greer, 2009; Taylor, DeQuinzio, \& Stine, 2012). Todos avaliaram o efeito do treino de respostas de monitoramento do comportamento de outros indivíduos (os participantes eram ensinados a discriminar respostas corretas e incorretas emitidas por outros) sobre a aprendizagem observacional de repertórios verbais, em situação ao vivo.
DeQuinzio e Taylor (2015) ensinaram quatro crianças com autismo a discriminarem respostas reforçadas e não reforçadas de um modelo adulto. As crianças aprenderam a imitar as nomeações de figuras pelo modelo em que eram reforçadas e a responderem "eu não sei" para nomeações de figura pelo modelo em que não eram reforçadas. Nos testes sem a presença do modelo, verificou-se que as crianças aprenderam a responder adequadamente às figuras utilizadas no treino, mas apresentaram desempenho variável com novas figuras.

Taylor et al. (2012) investigaram, em três crianças com autismo, o efeito do treino de respostas de monitoramento sobre a aprendizagem observacional de leitura de palavras (comportamento textual - Skinner, 1957/1992). As crianças foram expostas a duas condições experimentais alternadas: (1) treino de monitoramento das respostas textuais de um modelo, durante o qual as crianças observavam a resposta textual do modelo, respondiam à pergunta "o que ele disse?" imitando vocalmente a resposta do modelo e, em seguida, indicavam, em um arranjo de cinco palavras escritas, a que foi lida pelo modelo; e (2) apenas exposição às respostas textuais do modelo. Dez minutos após cada sessão de uma condição, era realizado um teste de leitura das palavras usadas na sessão. Os resultados mostraram que as crianças leram as palavras de maneira mais acurada quando as respostas de monitoramento foram adquiridas. No entanto, os autores concluíram que não foi possível determinar que aspecto da resposta de monitoramento facilitou a aquisição do comportamento textual.

O estudo de Pereira-Delgado e Greer (2009) buscou investigar se um treino de monitoramento de respostas corretas e incorretas do modelo poderia facilitar a aquisição de aprendizagem observacional. Esses autores verificaram, em um primeiro experimento, que duas crianças com autismo não apresentavam, em testes pré-experimentais, o repertório de aprendizagem observacional da leitura de palavras e nomeação de figuras (os operantes verbais textual e tato, respectivamente - Skinner, 1957/1992). Em seguida, as crianças foram treinadas a monitorar as respostas do modelo em situação ao vivo, respondendo discriminadamente às respostas textuais corretas e incorretas do modelo. $\mathrm{O}$ treino de monitoramento de respostas foi realizado em três etapas: Etapa 1 - o participante era ensinado a responder discriminadamente (por meio de cartões verde e vermelho) após observar a consequenciação das respostas textuais corretas e incorretas do modelo a estímulos conhecidos pelo participante; Etapa 2- como na Etapa 1, mas o participante tinha a oportunidade de responder discriminadamente antes que o pesquisador consequenciasse a resposta do modelo; Etapa 3 - semelhante à Etapa 1, mas o participante não conhecia (emitia) o textual_exigido e respondia após o pesquisador ter consequenciado a resposta do modelo. A aprendizagem observacional foi sondada após cada etapa por meio de testes idênticos ao pré-teste. As duas crianças 
mostraram aprendizagem observacional de textuais e tatos após a conclusão das três etapas do treino de monitoramento.

Pereira-Delgado e Greer (2009) realizaram um segundo experimento com três crianças (as duas do Experimento 1 e uma terceira também com atraso no desenvolvimento), replicando o procedimento do primeiro experimento com as seguintes alterações: (1) foi avaliada a aprendizagem observacional de soletração de palavras ditadas e (2) foram eliminados os testes após a conclusão de cada etapa de treino da resposta de monitoramento (foram realizados somente testes pré e pós-experimentais). As crianças não apresentaram aprendizagem observacional nos testes préexperimentais, tiveram uma rápida aquisição da resposta de monitoramento e mostraram aprendizagem observacional nos testes pós-experimentais.

Em resumo, alguns estudos (DeQuinzio \& Taylor, 2015; Pereira-Delgado \& Greer, 2009; Taylor et al., 2012) têm mostrado que o ensino do repertório de monitoramento do comportamento do modelo pode ser importante para a aprendizagem observacional em crianças com autismo. Em todos esses estudos, o ensino do repertório de monitoramento foi feito em situações "ao vivo". No entanto, conforme mencionado anteriormente, pessoas com autismo parecem se beneficiar mais da aprendizagem observacional implementada por meio de videomodelação (Bellini \& Akullian, 2007; Charlop-Christy et al., 2000; Nadel et al., 2011). Considerando isso, o presente estudo realizou uma replicação sistemática do Experimento 2 de Pereira-Delgado e Greer (2009) com as seguintes alterações: (1) pré e pós-testes de aprendizagem observacional de tatos e textuais via videomodelação e (2) treino de monitoramento do ensino de tatos para um modelo via videomodelação. Com a replicação, buscou-se investigar: (1) a possibilidade de estabelecer, em crianças com autismo, via videomodelação, o repertório de monitoramento do comportamento de outros indivíduos e (2) os efeitos da possível aquisição desse repertório sobre a aprendizagem via videomodelação de tatos e textuais.

\section{MÉTODO}

\section{Participantes}

Participaram do estudo duas crianças diagnosticadas com transtorno do espectro autista, Matheus e Henrique (nomes fictícios), de sete e três anos de idade, respectivamente. Elas estavam inseridas no projeto APRENDE (Atendimento e Pesquisa sobre Aprendizagem e Desenvolvimento; Barros, Souza, \& Assis, 2012), da Universidade Federal do Pará e frequentavam a escola regular. Seus repertórios verbais foram avaliados por meio do Verbal Behavior Milestones Assessment and Placement Program (VB-MAPP; Sundberg, 2008). As duas crianças alcançaram o nível 1 do VB-MAPP (0 a 18 meses) e completaram algumas habilidades do nível 2 (18 a 30 meses). Ambos não apresentavam textual de palavras e tinham repertório limitado de tatos.

O presente trabalho foi aprovado pelo Comitê de Ética em Pesquisa do Instituto de Ciências da Saúde da UFPA, conforme o parecer $n^{\circ} 175.303$ de 14/12/2012, em consonância com a Resolução 466/2012 do CNS/MS.

\section{Ambiente, Material e Equipamentos}

As sessões experimentais foram realizadas em uma sala de $2 \mathrm{~m}^{2}$ do APRENDE, climatizada e com iluminação artificial. A sala estava equipada com dois computadores HP All in One TouchSmart 20", modelo 310-1110br, com tela sensível ao toque, sendo um deles utilizado nas sessões de videomodelação. Um cartão vermelho e um verde (10 $\mathrm{cm} \times 10 \mathrm{~cm}$ ) foram empregados no treino de respostas de monitoramento. As respostas dos participantes foram registradas em folhas de registro específicas para o estudo e filmadas com uma câmera Sony HDD DCR-SR87.

\section{Estímulos Discriminativos}

Nos testes pré e pós-experimentais, foram utilizados quatro estímulos tridimensionais antropomórficos, nomeados com pseudo-palavras trissílabas (ver Figura 1) e quatro pseudo-palavras em formato escrito. Dessa forma, tanto nos pré e pós-testes de tato, quanto de textual, garantiase que os participantes não conheciam os estímulos e nem tinham contato com esses fora do laboratório. No treino de monitoramento, foram utilizados, como estímulos, objetos tridimensionais comuns do dia-a-dia, sendo oito objetos para os quais a criança emitia o tato (usados nas etapas 1 e 2 do estudo - ver Procedimento) e quatro objetos para os quais ela não emitia o tato (usados na Etapa 3 - ver Procedimento) (ver Tabela 1).

\section{Estímulos Consequenciadores}

Primeiramente, foi feito um levantamento de possíveis reforçadores baseado na indicação dos cuidadores dos participantes. Além disso, foram realizados testes de preferência para construção dos vídeos, antes das sessões dos pré e pós-testes e das sessões de treino de resposta de monitoramento, buscando garantir a efetividade reforçadora dos estímulos selecionados para cada criança (e.g., comestível, computador, tablet etc.). O teste consistia na apresentação simultânea de três estímulos, sendo que 


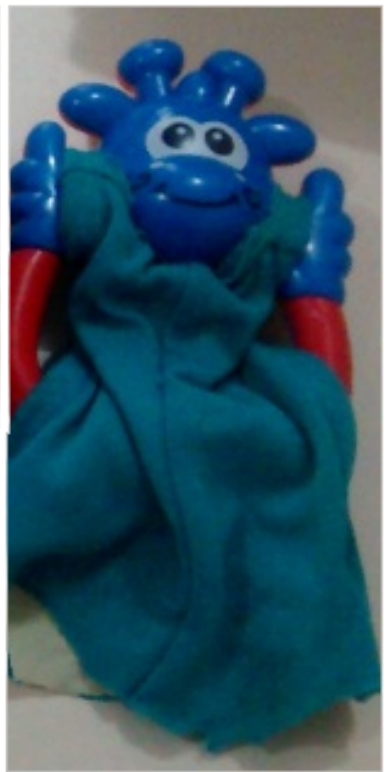

Cadoma

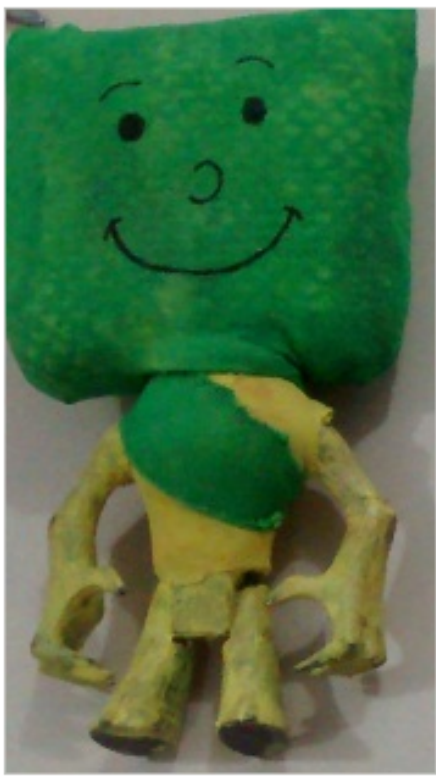

Sadebi

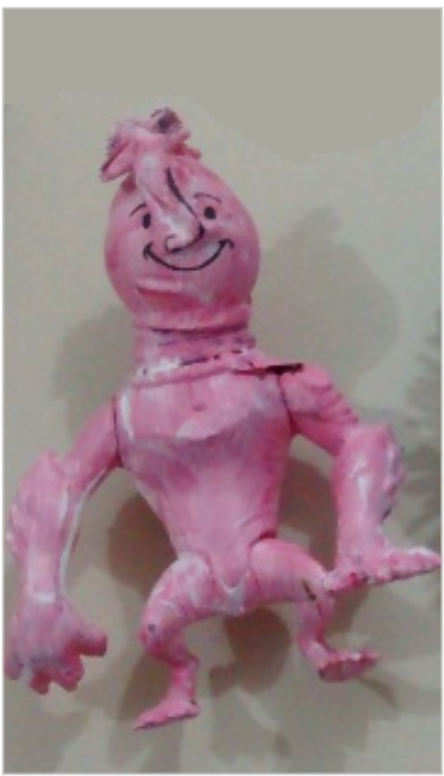

Tudelu

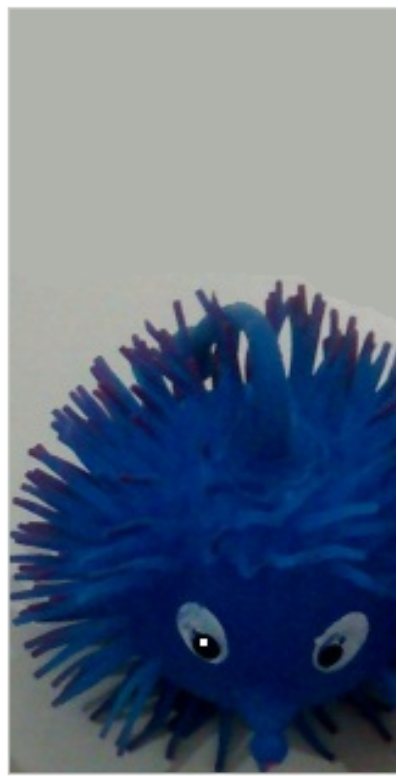

Lofapi

Figura 1. Estímulos antropomórficos e seus respectivos pseudo-nomes utilizados nos pré e pós-testes.

Tabela 1 Lista de estímulos utilizados nos pré e pós-testes, nas Etapas 1 a 3, e o tipo de apresentação no vídeo.

\begin{tabular}{|c|c|c|c|c|c|}
\hline Participante & Pré e Pós testes & Etapa 1 & Etapa 2 & Etapa 3 & Tipo de apresentação \\
\hline \multirow{4}{*}{ Matheus } & Lofapi & Balão & Bola & Tesoura & $100 \%$ correta \\
\hline & Cadoma & Telefone & Maçã & Teclado & $100 \%$ incorreta \\
\hline & Tudelu & Carro & Sapato & Grampeador & $50 \%$ correta/incorreta \\
\hline & Sadebi & Faca & Banana & Xícara & $50 \%$ incorreta/correta \\
\hline \multirow{4}{*}{ Henrique } & Sadebi & Balão & Maçã & Teclado & $100 \%$ correta \\
\hline & Cadoma & Tesoura & Cola & Furador & $100 \%$ incorreta \\
\hline & Tudelu & Banana & Garrafa & Xícara & $50 \%$ correta/incorreta \\
\hline & Lofapi & Carro & Câmera & Grampeador & $50 \%$ incorreta/correta \\
\hline
\end{tabular}

a criança deveria selecionar um a um os itens preferidos, sendo removido das opções o item escolhido, até que restasse apenas um. Posteriormente, os estímulos eram classificados de acordo com a ordem de escolha da criança e o item mais escolhido recebia a maior pontuação (adaptado de Carr, Nicolson, \& Higbee, 2000). Também foram utilizadas consequências sociais, como elogios (e.g., "Muito bem!", “Legal!").

\section{Procedimento}

Foi realizado um delineamento de pré e pós-testes com medidas repetidas. A variável dependente era a aprendizagem observacional, medida pela aquisição de textuais e tatos via videomodelação. A variável independente foi a implementação de um treino de monitoramento, de tatos corretos e incorretos, de um Modelo via videomodelação. Todas as sessões do experimento foram filmadas.

Avaliação da articulação de sílabas. Inicialmente, foi realizada uma avaliação de quais sílabas as crianças eram capazes de emitir. Uma sessão de avaliação consistiu em 20 tentativas, nas quais o pesquisador solicitava que a criança ecoasse sílabas (e.g., BA). Caso a criança respondesse adequadamente, recebia um dos reforçadores que já haviam sido identificados como reforçadores potenciais para os participantes (e.g., tablet), juntamente com consequência social (elogio, sorriso, etc.). Caso errasse ou não emitisse a resposta em $5 \mathrm{~s}$, o pesquisador passava para a tentativa seguinte. Foram realizadas sessões até se obter pelo menos 10 sílabas que a criança reproduzisse corretamente. Essas sílabas foram, então, utilizadas para compor os nomes dos estímulos antropomórficos e as pseudo-palavras.

Seleção de objetos tateados e não tateados. A seleção de objetos do dia-a-dia que as crianças tateavam e que não tateavam foi realizada em sessões de 20 tentativas com 20 objetos do cotidiano, apresentados de forma randomizada. No início de cada tentativa, o objeto era apresentado e o pesquisador perguntava "o que é isso?" ou "qual o nome dele?". A criança tinha $5 \mathrm{~s}$ para responder à pergunta. Não havia consequência programada para respostas corretas ou incorretas (respostas parciais e não emissão de resposta) e, em seguida, iniciava-se a próxima tentativa. A cada três 
tentativas, era solicitado um comportamento alternativo presente no repertório da criança (e.g., "toque aqui"), o qual era seguido de reforços tangíveis e sociais, a fim de manter o engajamento da criança na tarefa. Foram selecionados, para cada participante, oito objetos tateados e quatro não tateados (ver Tabela 1).

Elaboração dos vídeos. Os vídeos dos testes foram elaborados após a definição dos nomes dos estímulos antropomórficos, das palavras escritas e do treino, uma vez feito o levantamento de tatos conhecidos e desconhecidos pelo participante. A filmagem foi feita em uma perspectiva de terceira pessoa, ou seja, como se outra pessoa estivesse vendo uma criança (modelo) e um adulto (pesquisador), com o intuito de torná-la o mais próximo possível da situação 'ao vivo' do estudo de Pereira-Delgado e Greer (2009). Em cada vídeo, o pesquisador, sentado de frente para o modelo, apresentava o estímulo para esse e perguntava " $O$ que é isso?"/ "o que está escrito", sendo focados o modelo e estímulo apresentado.

Para os testes pré e pós-experimentais de aprendizagem observacional de tatos, foram filmadas cenas em que o pesquisador ensinava o modelo a nomear objetos tridimensionais antropomórficos. Os nomes dos objetos eram pseudo-palavras trissílabas. No vídeo, o pesquisador apresentava o estímulo para o modelo e perguntava "O que é isso?", sendo focados o modelo e o objeto na mão do pesquisador. Foram filmadas cenas nas quais o modelo respondia corretamente e cenas nas quais ele respondia incorretamente. Nas cenas de respostas corretas, o modelo emitia o tato e, em seguida, tinha acesso a um dos reforçadores tangíveis e a uma consequência social (elogio, sorriso, etc.). Nas cenas de respostas incorretas, o modelo não emitia o tato correspondente e o pesquisador respondia "Não, isso é X". O modelo, então, ecoava o nome do objeto, recebendo, em seguida, reforço social.

Para os testes pré e pós-experimentais de aprendizagem observacional de comportamento textual, os vídeos mostravam cenas em que o modelo aprendia pseudopalavras. As cenas seguiram o mesmo padrão do vídeo de teste de tato, a única diferença era que o pesquisador apresentava uma pseudo-palavra escrita e perguntava "O que está escrito?" à criança, sendo focados o modelo e a tela do computador na qual aparecia a pseudo-palavra. Além disso, a criança emitia o textual correto ou incorreto, com correção.

Os vídeos do treino de monitoramento eram semelhantes aos dos testes de aprendizagem observacional, excetuandose que o pesquisador apresentava, nas Etapas 1 e 2, objetos do dia-a-dia que a criança já nomeava corretamente. Além disso, foram filmadas tentativas com quatro objetos que $o$ participante não nomeava para a Etapa 3. Foram filmadas cenas tanto com tentativas corretas, quanto tentativas incorretas com correção e a consequência para o modelo seguiu o formato dos vídeos anteriores.

De maneira diferente do que foi feito por Pereira-Delgado e Greer (2009), as cenas dos vídeos foram apresentadas de forma randomizada ao longo das sessões de teste de aprendizagem observacional e de treino de monitoramento. De forma a tornar equilibradas as apresentações das tentativas, foram utilizados quatro estímulos, dos quais: um deles foi apresentado sempre em tentativas corretas; um segundo estímulo foi apresentado sempre em tentativas incorretas com correção e os outros dois foram apresentados em metade das tentativas corretas e incorretas com correção (Tabela 1).

Pré-teste de aprendizagem observacional. Os participantes passaram por sessões de testes do repertório de aprendizagem observacional de tatos e textuais de estímulos tridimensionais arbitrários. Para o pré-teste de tatos, cada sessão consistiu em 16 tentativas, randomicamente apresentadas, nas quais o participante observava os quatro estímulos serem tateados pelo modelo quatro vezes. Após quatro tentativas de observação do vídeo, uma com cada estímulo, era feito o teste no mesmo local. Nesse teste, o pesquisador perguntava "o que é isso?" para cada um dos quatro estímulos. A sequência observação-teste foi feita mais três vezes com os mesmos estímulos. Houve intervalos de 3 min entre as sequências observação-teste, durante os quais o pesquisador interagia com a criança e fazia demandas conhecidas por essa. Assim, como na seleção de tatos, a criança deveria responder em até $5 \mathrm{~s}$ após a pergunta do pesquisador. A resposta considerada adequada era o tato correto dos estímulos tateados pelo Modelo (e.g., "sadebi"). Considerou-se inadequada tanto uma resposta parcial, quanto a ausência de resposta. Não havia consequências programadas para respostas adequadas ou inadequadas. $\mathrm{O}$ critério para considerar que o participante apresentava o repertório foi de oito ou mais tentativas corretas.

O mesmo procedimento foi realizado no pré-teste de textuais, no qual foram observadas 16 tentativas totais de textuais. Cada pseudo-palavra foi apresentada na tela do computador, na fonte Times New Roman, regular, tamanho 96, em letras negras centralizadas em um fundo branco de um slide do programa PowerPoint. Após quatro tentativas de observação do vídeo, uma com cada palavra, foi feito o teste no mesmo local, o qual consistiu no pesquisador solicitando “o que está escrito?" ou "que palavra é essa?" para cada uma das quatro palavras escritas na tela do computador, apresentadas uma por vez. A sequência observação-teste e os critérios utilizados foram os mesmos do teste de tato.

Treino de monitoramento. Foi realizado um treino de monitoramento de tatos corretos e incorretos para os dois participantes. O treino foi dividido em três etapas: (1) instalação da resposta de monitoramento; (2) desenvolvimento do controle do responder do participante pelo responder do modelo; e (3) estabelecimento do controle do responder do participante pela consequência para o modelo. Foram realizadas de duas a três sessões semanais. Cada sessão compreendia quatro blocos de quatro tentativas, apresentadas de forma randômica, totalizando 16 tentativas por sessão. Todas as etapas tinham como critério 
de aprendizagem de $90 \%$ de acertos, em duas sessões consecutivas, ou uma sessão com $100 \%$ de acertos. O vídeo era apresentado na tela do computador por meio do programa Windows Media Player ${ }^{\circledR}$.

Etapa 1: Instalar a resposta de monitoramento. Foram utilizados quatro objetos que já evocavam resposta de tato do participante (ver Tabela 1), selecionados na fase de seleção de objetos tateados. Durante a tentativa, o pesquisador apresentava uma cena completa de videomodelação e, posteriormente, dava a oportunidade ao participante de avaliar a resposta do modelo, perguntando "o que aconteceu?". A resposta de avaliação requerida foi escolher o cartão verde ao observar respostas corretas do modelo, ou cartão vermelho para respostas incorretas.

A resposta de monitoramento foi treinada por meio de dois passos. No primeiro passo, o pesquisador fornecia a dica vocal "ele acertou/errou" e apontava para o cartão correto e, além disso, ajudava fisicamente a criança a apontar para o cartão correto. No segundo passo, após quatro tentativas, ocorria um atraso de $3 \mathrm{~s}$ no fornecimento da dica, buscando possibilitar a ocorrência de respostas independentes do participante.

A consequência para a resposta correta de monitoramento era a liberação de reforçadores tangíveis e consequências sociais. Respostas incorretas produziam a repetição da cena, fornecendo-se dica para a resposta correta, assim como foi feito no primeiro passo, seguida de consequência social e apresentação da próxima tentativa.

Etapa 2: Desenvolver o controle do responder do participante pelo responder do modelo. Foram utilizados outros quatro objetos que já evocavam a resposta de tato dos participantes (ver Tabela 1). Essa etapa diferenciou-se da Etapa 1 pela oportunidade do participante monitorar a resposta do modelo antes que a consequência fosse apresentada no vídeo. Ou seja, o pesquisador apresentava a cena em vídeo, porém a interrompia antes que a resposta do modelo fosse consequenciada, exigindo uma resposta (apresentação do cartão vermelho ou verde) do participante. Logo após a resposta, o pesquisador liberava consequências de forma semelhante ao que foi feito na Etapa 1 e apresentava o final do vídeo dizendo "Você acertou" (para respostas de monitoramento corretas) ou "Não, essa não é a resposta. Vamos ver novamente?" (para respostas de monitoramento incorretas).

Etapa 3: Estabelecer controle do responder do participante pela consequência para o modelo. Esse treino era semelhante ao da Etapa 1, diferenciando-se pelo fato de que os objetos utilizados reconhecidamente não controlavam o repertório de tato requerido. $\mathrm{O}$ pesquisador apresentava a cena completa em vídeo e, em seguida, fornecia a oportunidade ao participante de monitorar a resposta do modelo. A consequência era semelhante àquela da Etapa 1 .

Pós-testes de aprendizagem observacional. Os pós-testes foram idênticos aos pré-testes de aprendizagem observacional de tatos e textuais via videomodelação, porém, acrescentou-se outro teste de tato com os objetos que haviam aparecido nas cenas de vídeo da Etapa 3 do treino de monitoramento.

\section{REGISTRO E ANÁLISE DE DADOS}

Foram registrados, para cada sessão, o percentual de acertos para cada estímulo e para o conjunto de quatro estímulos. Durante as sessões experimentais, o pesquisador registrava as respostas dos participantes, para cada tentativa, em uma folha de registro específica. Posteriormente, outro pesquisador fez o registro de $30 \%$ do total de sessões (selecionadas de cada fase), para (1) estabelecer um índice de concordância entre observadores do registro das respostas dos participantes ([Concordância/Concordância + Discordância] x 100) e (2) avaliar a integridade do procedimento. Nesse último caso, era verificado se os procedimentos planejados (apresentação dos vídeos para cada fase do procedimento, apresentação dos estímulos, consequências etc.) foram implementados corretamente para cada participante ([Implementações corretas/ Total de Implementações] x 100). A concordância entre observadores do registro do desempenho dos dois participantes foi de $95 \%$ para cada. A análise da integridade do procedimento quanto à apresentação dos vídeos para cada fase foi de $100 \%$ para os dois participantes, e também de 100\% quanto à apresentação dos estímulos e consequências para os dois participantes.

\section{RESULTADOS}

A Figura 2 apresenta a porcentagem de acertos por sessão dos participantes nas fases de pré-testes de aprendizagem observacional de tato e textual, treino de monitoramento e pós-testes de aprendizagem observacional de tatos e textual.

Nos pré-testes de aprendizagem observacional, o participante Matheus alcançou, no máximo, 25\% de acertos nas três sessões de aprendizagem observacional de tato.
Todos os acertos foram com o estímulo Cadoma (ver Tabela 2, que apresenta o percentual de acertos por estímulos nos pré-testes e pós-testes de aprendizagem observacional de tato e textual para Matheus). Não houve acertos na sessão de aprendizagem observacional de textuais. O participante Henrique apresentou, no máximo, $12,5 \%$ de acertos nas quatro sessões de aprendizagem observacional de tato e 


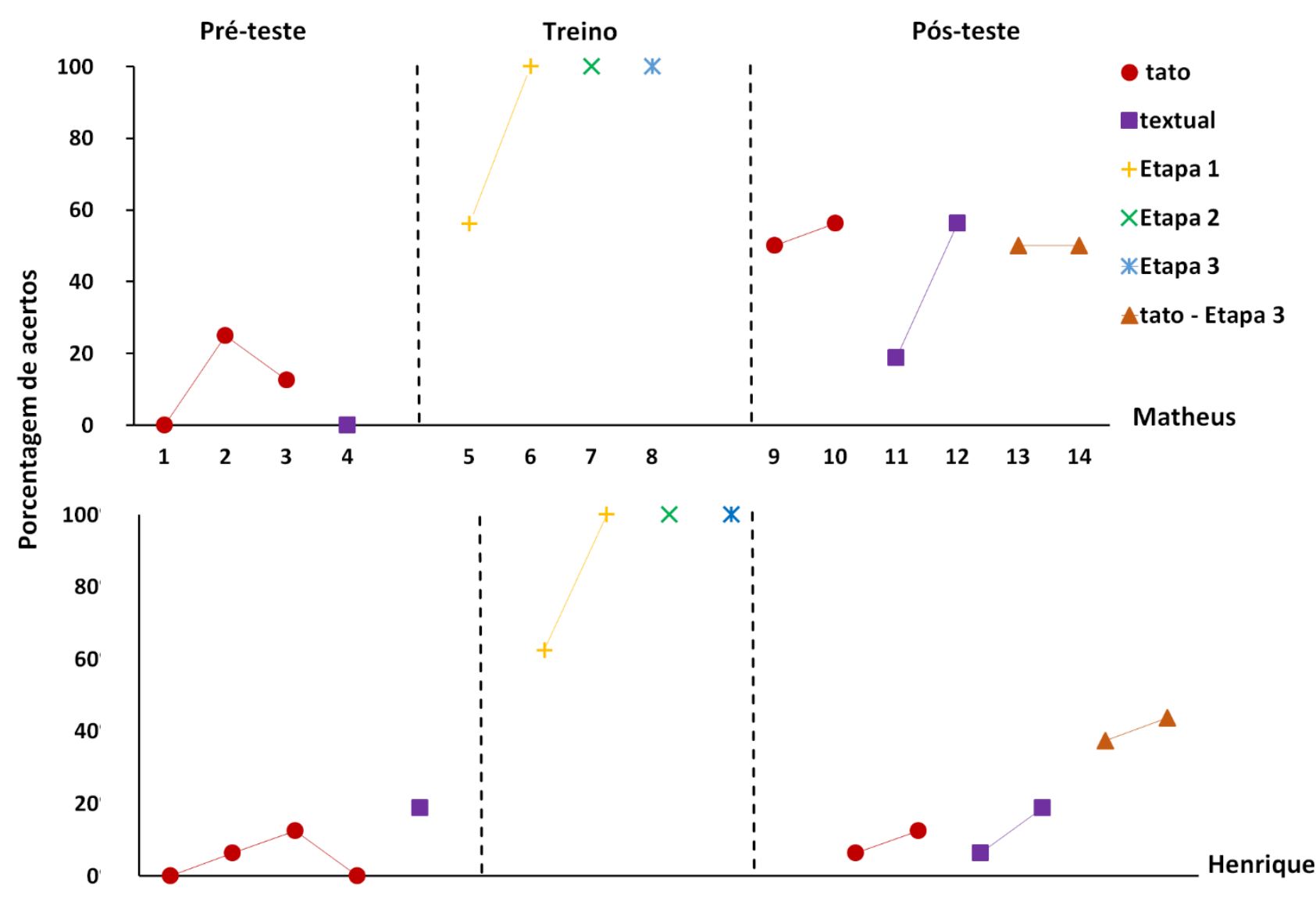

Figura 2. Porcentagem de acertos, por sessão, dos participantes Matheus e Henrique nos pré-testes de aprendizagem observacional (tato e textual), no treino de monitoramento e nos pós-testes de aprendizagem observacional (tato, textual e novos tatos, apresentados na Etapa 3 de monitoramento).

Tabela 2 Percentual de acertos por estímulos nos pré e pós-testes de aprendizagem observacional de tato e textual para o participante Matheus.

\begin{tabular}{|c|c|c|c|c|c|c|c|c|}
\hline \multirow{4}{*}{ Estímulos } & \multicolumn{4}{|c|}{ PRÉ-TESTES } & \multicolumn{4}{|c|}{ PÓS-TESTES } \\
\hline & \multicolumn{3}{|c|}{ TATO } & \multirow{2}{*}{$\begin{array}{c}\text { TEXTUAL } \\
\text { Sessão }\end{array}$} & \multicolumn{2}{|c|}{ TATO } & \multicolumn{2}{|c|}{ TEXTUAL } \\
\hline & \multicolumn{3}{|c|}{ Sessão } & & \multicolumn{2}{|c|}{ Sessão } & \multicolumn{2}{|c|}{ Sessão } \\
\hline & 1 & 2 & 3 & 1 & 1 & 2 & 1 & 2 \\
\hline Cadoma & 0 & 100 & 50 & 0 & 100 & 100 & 75 & 100 \\
\hline Lofapi & 0 & 0 & 0 & 0 & 0 & 0 & 0 & 0 \\
\hline Tudelu & 0 & 0 & 0 & 0 & 100 & 100 & 0 & 100 \\
\hline Sadebi & 0 & 0 & 0 & 0 & 0 & 0 & 0 & 25 \\
\hline Tesoura* & - & - & - & - & 100 & 100 & - & - \\
\hline Grampeador* & - & - & - & - & 0 & 0 & - & - \\
\hline Xícara* & - & - & - & - & 100 & 100 & - & - \\
\hline Teclado* & - & - & - & - & 0 & 0 & - & - \\
\hline
\end{tabular}

*Estímulos utilizados na Etapa 3 do treino de monitoramento. 
$18,75 \%$ de acertos na sessão de aprendizagem observacional de textual. Todos os acertos foram também com o estímulo Cadoma (ver Tabela 3, que apresenta o percentual de acertos por estímulos nos pré-testes e pós-testes de aprendizagem observacional de tato e textual para Henrique).

No treino de monitoramento, ambos os participantes alcançaram o critério de aprendizagem para a Etapa $1 \mathrm{em}$ duas sessões, com $60 \%$ de acertos na primeira sessão e $100 \%$ de acerto na segunda. Além disso, os dois participantes alcançaram o critério de aprendizagem em apenas uma sessão nas etapas 2 e 3, com $100 \%$ de acerto.

No pós-teste de aprendizagem observacional de tato, Matheus acertou $50 \%$ das tentativas na primeira sessão e $56,25 \%$ na segunda sessão, verificando-se um aumento médio de $41 \%$ de acertos com relação ao pré-teste. Cabe destacar que Matheus apresentou $100 \%$ de acertos para dois dos quatro estímulos (Cadoma e Tudelu; ver Tabela 2). No pós-teste de textual, Matheus obteve $18,75 \%$ de acertos na primeira sessão e $56,25 \%$ na segunda sessão. Na segunda sessão, Matheus acertou todas as tentativas para dois estímulos (Cadoma e Tudelu) e uma para Sadebi (ver Tabela 2).

Matheus acertou 50\% das tentativas nas duas sessões de pós-teste com os estímulos da Etapa 3. Ele apresentou 100\% de acertos para dois estímulos (Tesoura e Xícara) nas duas sessões (ver Tabela 2).
O participante Henrique apresentou 6,25\% de acertos na primeira sessão de pós-testes de tato, e 12,5\% na segunda sessão. Todos os acertos foram com o estímulo Sadebi (ver Tabela 3). No pós-teste de textual, Henrique obteve $6,25 \%$ de acertos na primeira sessão e $18,75 \%$ na segunda. Os acertos foram com os estímulos Sadebi e Cadoma (ver Tabela 3). No pós-teste com os estímulos da Etapa 3, Henrique apresentou $37,5 \%$ de acertos na primeira sessão e $43,75 \%$ na segunda sessão. Ele obteve $100 \%$ de acertos para um estímulo (Xícara) nas duas sessões, e 50\% para o estímulo Grampeador na segunda sessão (ver Tabela 3).

Não houve influência da forma de apresentação dos vídeos, com tentativas sempre corretas, sempre incorretas com correção ou ambas as formas, na aquisição de tatos e textuais via videomodelação. Como pode ser visto nas tabelas 1, 2 e 3, o participante Matheus adquiriu tato e textual para o estímulo que foi apresentado sempre de forma incorreta com correção (Cadoma) e para o que foi apresentado de ambas as formas (Tudelu). Para os estímulos da Etapa 3, Matheus adquiriu tato para o estímulo que foi apresentado sempre de forma correta (Tesoura) e para um dos que foi apresentado de ambas as formas (Xícara), enquanto Henrique adquiriu o tato para um dos estímulos que foi apresentado de ambas as formas (Xícara).

Tabela 3 Percentual de acertos por estímulos nos pré e pós-testes de aprendizagem observacional de tato e textual para o participante Henrique.

\begin{tabular}{|c|c|c|c|c|c|c|c|c|c|}
\hline \multirow{4}{*}{ Estímulos } & \multicolumn{5}{|c|}{ PRÉ-TESTES } & \multicolumn{4}{|c|}{ PÓS-TESTES } \\
\hline & \multicolumn{4}{|c|}{ TATO } & \multirow{3}{*}{$\begin{array}{c}\text { TEXTUAL } \\
\text { Sessão } \\
1\end{array}$} & \multirow{2}{*}{\multicolumn{2}{|c|}{$\begin{array}{c}\text { TATO } \\
\text { Sessão }\end{array}$}} & \multirow{2}{*}{\multicolumn{2}{|c|}{$\begin{array}{c}\text { TEXTUAL } \\
\text { Sessão }\end{array}$}} \\
\hline & \multicolumn{4}{|c|}{ Sessão } & & & & & \\
\hline & 1 & 2 & 3 & 4 & & 1 & 2 & 1 & 2 \\
\hline Cadoma & 0 & 25 & 50 & 0 & 75 & 0 & 0 & 0 & 25 \\
\hline Lofapi & 0 & 0 & 0 & 0 & 0 & 0 & 0 & 0 & 0 \\
\hline Tudelu & 0 & 0 & 0 & 0 & 0 & 0 & 0 & 0 & 0 \\
\hline Sadebi & 0 & 0 & 0 & 0 & 0 & 25 & 50 & 25 & 50 \\
\hline Furador* & - & - & - & - & - & 25 & 25 & - & - \\
\hline Grampeador* & - & - & - & - & - & 25 & 0 & - & - \\
\hline Xícara* & - & - & - & - & - & 100 & 100 & - & - \\
\hline Teclado* & - & - & - & - & - & 0 & 50 & - & - \\
\hline
\end{tabular}

*Estímulos utilizados na Etapa 3 do treino de monitoramento

\section{DISCUSSÃO}

O presente estudo buscou ampliar a base experimental da investigação sobre as variáveis que afetam a aprendizagem observacional em pessoas com diagnóstico de Transtorno do Espectro Autista, tratando, dessa forma, esse repertório como variável dependende (DeQuinzio \& Taylor, 2015; Pereira-Delgado \& Greer, 2009; Taylor \& DeQuinzio, 2012). Foi realizada uma replicação sistemática do Experimento 2 de Pereira-Delgado e Greer (2009), com treino de monitoramento do ensino de tatos para um modelo via videomodelação e pré e pós-testes de aprendizagem observacional de tatos e textuais, também via videomodelação, considerando que a literatura tem sugerido que, em pessoas com esse diagnóstico, a aprendizagem observacional pode ser favorecida por meio de videomodelação (Charlop-Christy et al., 2000; Nadel et al., 2011).

Os resultados mostraram que crianças com autismo podem aprender em poucas tentativas de treino, via 
videomodelação, o repertório de monitoramento do comportamento de outros indivíduos. Comparando a aprendizagem do repertório de monitoramento no presente estudo com a do estudo de Pereira-Delgado e Greer (2009), observa-se que o treino via videomodelação se mostrou mais eficiente, uma vez que, no presente estudo, foram necessárias 56 e 57 tentativas para os participantes finalizarem o treino, enquanto que, no estudo de Pereira-Delgado e Greer (2009), foram realizadas 180 a 220 tentativas para o treino de monitoramento "ao vivo". Esse resultado reforça os dados da literatura que apontam um efeito facilitador do treino via videomodelação no ensino de repertórios para pessoas com autismo (Bellini \& Akullian, 2007; Charlop-Christy et al., 2000; Cobertt \& Abdullah, 2005; Nadel et al., 2011; Plavnick \& Ferreri, 2011).

Por outro lado, no estudo atual, a aquisição do repertório de monitoramento das respostas de outro indivíduo favoreceu apenas parcialmente a aquisição de tatos e textuais por aprendizagem observacional e para somente um dos dois participantes (Matheus - que aprendeu dois dos quatro tatos e um dos textuais avaliados no pré-teste e dois dos quatro tatos utilizados na Etapa 3 do treino de monitoramento). No estudo de Pereira-Delgado e Greer (2009), o treino de monitoramento favoreceu a aprendizagem observacional para os três participantes. No entanto, além da diferença no número de tentativas para finalizar o treino de monitoramento, deve-se ressaltar que, no estudo desses autores, foram realizadas repetidas sessões de 20 tentativas nos pós-testes até que os participantes alcançassem o critério de $90 \%$ de aprendizagem do repertório, de forma que os participantes realizaram de 100 a 140 tentativas de pós-teste. Essas tentativas, somadas às tentativas de préteste, totalizaram de 220 a 280 tentativas de exposição aos estímulos a serem aprendidos via observação. No presente estudo, foram realizadas apenas duas sessões de 16 tentativas nos pós-testes de aprendizagem observacional para cada repertório testado - tato, textual e tato dos estímulos utilizados na Etapa 3 do treino. Essas tentivas, somadas às tentativas de pré-teste de cada repertório ou do treino da Etapa 3, totalizaram de 80 a 96 tentativas de exposição aos tatos a serem aprendidos via videomodelação e 48 tentativas de exposição aos textuais e tatos do treino da Etapa 3.

Tanto no estudo de Pereira-Delgado e Greer (2009), como no presente estudo, as tentativas de pré e pósteste consistiram em situações ("ao vivo" ou filmadas, respectivamente) nas quais os participantes eram expostos a outros indivíduos, respondendo corretamente (ou incorretamente com correção) aos estímulos apresentados. Nessas situações, cada tentativa correspondia a um pareamento entre o estímulo e a resposta correta para ele, o que vem sendo caracterizado como procedimento de observação de pareamento de estímulos (stimulus pairing observation procedure - Byrne, Rehfeldt, \& Aguirre, 2014). Esse procedimento tem sido eficaz para ensinar repertórios verbais para crianças com desenvolvimento típico e atípico sem treinos com consequenciação diferenciada de respostas (Carnerero \& Pérez-González, 2014; Rosales, Rehfeldt, \& Huffman, 2012), sendo a frequência dos pareamentos uma variável relevante para a aprendizagem. Considerando-se essa literatura e os resultados dos pré e pós-testes do estudo de Pereira-Delgado (2009) e do presente estudo, supõe-se que uma maior exposição aos pré e pós-testes no atual estudo poderia ter favorecido a aquisição observacional dos tatos e textuais apresentados. Estudos futuros podem avaliar essa questão utilizando um delineamento de linha de base múltipla entre participantes e medidas repetidas de pós-teste. Deve ser avaliado, também, o efeito de uma maior exposição ao treino de monitoramento na aprendizagem via videomodelação de repertórios verbais em crianças com autismo.

Deve-se destacar que, ainda que os participantes tivessem repertórios verbais semelhantes no início do estudo atual (ausência de textual e tato limitado), o desempenho do participante que tinha três anos de idade (Henrique) pode ter sido especialmente afetado pela demanda das tarefas (aprendizagem via videomodelação de tatos e, especialmente, de textuais) e pela diferença que havia entre $o$ treino da resposta de monitoramento, que era feito com tatos do modelo e as avaliações de pré e pós-teste que exigiam a aprendizagem observacional de tatos e textuais do modelo. A diferença na idade cronológica dos participantes (Henrique três anos; Matheus - sete anos) pode ter implicado, também, diferentes repertórios pré-requisitos para o monitoramento de respostas de outros e aprendizagem observacional, tais como imitação, condicionamento das ações de outros indivíduos como estímulos discriminativos/reforçadores e atenção compartilhada (MacDonald \& Ahearn, 2015; Pereira-Delgado \& Greer, 2009; Plavnick \& Hume, 2014), que não foram avaliados neste estudo. Essas limitações do presente estudo podem ser corrigidas em pesquisas futuras com a realização de pré-testes e treinos, se necessário, de alguns dos repertórios apontados como pré-requisitos para o monitoramento de respostas de outros e aprendizagem observacional, a seleção de tarefas com demandas mais ajustadas às idades cronológicas dos participantes e menor discrepância entre as situações de treino e testes.

Com os cuidados metodológicos mencionados acima e considerando o bom resultado no treino de monitoramento via videomodelação no presente estudo, o desenvolvimento da linha de pesquisa, com interesse nas variáveis relevantes para a aprendizagem observacional, pode investigar quais variáveis do treino de monitoramento via videomodelação poderiam produzir melhor desempenho na aquisição do repertório de aprendizagem observacional via videomodelação em crianças com autismo. Nessa direção, podem ser realizados tratamentos nos vídeos (uso de recursos para realçar os estímulos e as respostas do modelo), testes após cada cena e não após a observação de todas as cenas, a fim de reduzir a complexidade discriminativa nos treinos e testes e uma investigação paramétrica do intervalo mais 
adequado entre a exposição ao(s) estímulo(s) e os testes relacionados a ele(s).

Além disso, considerando o caráter translacional do tipo de investigação como a do presente estudo, novas pesquisas sobre os efeitos da aquisição de resposta de monitoramento via videomodelação para a aprendizagem observacional devem buscar garantir procedimentos de treino que favoreçam a generalização do repertório aprendido (Stokes \& Baer, 1977). Podem ser realizados, por exemplo, treinos de monitoramento com múltiplos exemplares de estímulos e diferentes Modelos e testes via videomodelação e em situações "ao vivo".

\section{Agradecimentos}

Esta pesquisa contou com financiamento no âmbito do Instituto Nacional de Ciência e Tecnologia sobre Comportamento, Cognição e Ensino (CNPq \#465686/2014-1 e FAPESP \#2014/50909-8). ICSB recebeu bolsa de mestrado do CNPq e RBS e CBAS bolsa de produtividade do CNPq.

\section{REFERÊNCIAS}

American Psychiatric Association. (2013). Diagnostic and statistical manual of mental disorders (5th ed.). Arlington, VA: American Psychiatric Publishing.

Ayres, K. M., \& Langone, J. (2005). Intervention and instruction with video for students with autism: A review of the literature. Education and Training in Developmental Disabilities, 40(2), 183-196.

Bandura, A. (1979). Modificação do comportamento (E. Nick \& L. Peota, Trad.). Rio de Janeiro: Interamericana (Trabalho original publicado em 1969).

Barros, R. S., Souza, C. B. A., \& Assis, G. (2012). APRENDE: Atendimento e pesquisa sobre aprendizagem e desenvolvimento (Projeto de pesquisa não-publicado). Universidade Federal do Pará, Belém, Pará, Brasil.

Bellini, S., \& Akullian, J. (2007). A meta-analysis of video modeling and video self-modeling interventions for children and adolescents with autism spectrum disorders. Exceptional Children, 73(3), 264-287. doi: 10.1177/001440290707300301

Byrne, B. L., Rehfeldt, R. A., \& Aguirre, A. A. (2014). Evaluating the effectiveness of the stimulus pairing observation procedure and multiple exemplar instruction on tact and listener responses in children with autism. The Analysis of Verbal Behavior, 30 (2), 160-169. doi: 10.1007/s40616-014-0020-0

Carnerero, J. J., \& Pérez-González, L. A. (2014). Induction of naming after observing visual stimuli and their names in children with autism. Research in Developmental Disabilities, 35(10), 2514-2526. doi: 10.1016/j.ridd.2014.06.004

Carr, J. E., Nicolson, A. C., \& Higbee, T. S. (2000). Evaluation of a brief multiple-stimulus preference assessment in a naturalistic context. Journal of Applied Behavior Analysis, 33(3), 353-357. doi: 10.1901/jaba.2000.33-353

Charlop, M. H., Dennis, B., Carpenter, M. H., \& Greenberg, A. L. (2010). Teaching socially expressive behaviors to children with autism through video modeling. Education and Treatment of Children, 33(3), 371-393.

Charlop-Christy, M. H., Le, L., \& Freeman, K. A. (2000). A comparison of video modeling with in vivo modeling for teaching children with autism. Journal of Autism and Developmental Disorders, 30(6), 537-552.

Corbett, B. A., \& Abdullah, M. (2005). Video modeling: Why does it work for children with autism? Journal of Early and Intensive Behavior Intervention, 2(1), 2-8. doi: 10.1037/h0100294

Delano, M. E. (2007). Video modelling interventions for individuals with autism. Remedial and Special Education, 28(1), 33-42. doi: 10.1177/07419325070280010401

DeQuinzio, J. A., \& Taylor, B. A. (2015). Teaching children with autism to discriminate the reinforced and nonreinforced responses of others: Implications for observational learning. Journal of Applied Behavior Analysis, 48(1), 38-51. doi: 10.1002/jaba.192.
Fryling, M. J., Johnston, C., \& Hayes, L. J. (2011). Understanding observational learning: An interbehavioral approach. The Analysis of Verbal Behavior, 27(1), 191-203.

Gena, A. Couloura, S., \& Kymissis, E. (2005). Modifying the affective behavior of preschoolers with autism using in-vivo or video modeling and reinforcement contingencies. Journal of Autism and Developmental Disorders, 35(5), 545-556. doi: 10.1007/s10803-005-0014-9

Greer, R. D., Dudek-Singer, J., \& Gautreaux, G. (2006). Observational learning. International Journal of Psychology, 41(6), 486-499. doi: 10.1080/00207590500492435

Leaf, J. B., Oppenheim-Leaf, M. L., Leaf, R., Courtemanche, A. B., Taubman, M., Mceachin, J.,Sheldon, J. B., \& Sherman, J. A. (2012). Observational effects on the preferences of children with autism. Journal of Applied Behavior Analysis, 45(3), 473483. doi: 10.1901/jaba.2012.45-473

MacDonald, J., \& Ahearn W. H. (2015). Teaching observational learning to children. with autism. Journal of Applied Behavior Analysis, 48(4), 800-16. doi: 10.1002/jaba.257.

McCoy, K., \& Hermansen, E. (2007). Video modeling for individuals with autism: A review of model types and effects. Education and Treatment of Children, 30(4), 183-213.

Nadel, J., Aouka, N., Coulon, N., Gras-Vincendon, A., Canet, P., Fagard, J., \&, Bursztejn, C. (2011). Yes they can! An approach to observational learning in low-functioning children with autism. Autism, 15(4), 421-435. doi: 10.1177/1362361310386508

Nikopoulos, C. K., \& Keenan, M. (2007). Using video modeling to teach complex social sequences to children with autism. Journal of Autism and Developmental Disorder, 37(4), 678693. doi: 10.1007/s10803-006-0195-x

Pereira-Delgado, J. A., \& Greer, R. D. (2009). The effects of peer monitoring training on the emergence of the capability to learn from observing instruction received by peers. The Psychological Record, 59(3), 407-434.

Plavnick, J. B., \& Ferreri, S. J. (2011). Establishing verbal repertoires in children with autism using function-based video modeling. Journal of Applied Behavior Analysis, 44(4), 747766. doi: 10.1901/jaba.2011.44-747

Plavnick, J. B., \& Hume, K. A. (2014). Observational learning by individuals with autism: A review of teaching strategies. Autism: International Journal of Research and Practice, 18(4), 458-466. doi: 10.1177/1362361312474373

Rosales-Ruiz, J., \& Baer, D. M. (1997). Behavioral cusps: A developmental and pragmatic concept for Behavior Analysis. Journal of Applied Behavior Analysis, 30(3), 533-544. doi: 10.1901/jaba.1997.30-533

Rosales, R. Rehfeldt, R. A., \& Huffman, N. (2012). Examining the utility of the stimulus pairing observation procedure with preschool children learning a second language. Journal of Applied Behavior Analysis, 45(1), 173-175. doi: 10.1901/ 
jaba.2012.45-173

Skinner, B. F. (1992). Verbal behavior. Acton, MA: Copley Publishing Group. (Trabalho original publicado em 1957)

Stokes, T. F., \& Baer, D. M. (1977). An implicit technology of generalization. Journal of Applied Behavior Analysis, 10(2), 349-367. doi: 10.1901/jaba.1977.10-349

Sundberg, M. L. (2008) Verbal behavior milestones assessment and placement program: The VB-MAPP. Concord, CA: AVB Press.
Taylor, B. A., \& DeQuinzio, J. A. (2012). Observational learning and children with autism. Behavior Modification, 36(3), 341360. doi: $10.1177 / 0145445512443981$

Taylor, B. A., DeQuinzio, J. A., \& Stine, J. (2012). Increasing observational learning of children with autism: A preliminary analysis. Journal of Applied Behavior Analysis, 45(4), 815-820. doi: 10.1901/jaba.2012.45-815

Recebido em: 09/11/15

Primeira decisão editorial: 26/07/16

Aceito em: 17/10/16 